Relations industrielles

Industrial Relations

\title{
Alan M. Glassman and Thomas G. Cummings, Cases in Organizational Development
}

\section{Laurent Bélanger}

Volume 47, numéro 1, 1992

URI : https://id.erudit.org/iderudit/050756ar

DOI : https://doi.org/10.7202/050756ar

Aller au sommaire du numéro

Éditeur(s)

Département des relations industrielles de l'Université Laval

ISSN

0034-379X (imprimé)

1703-8138 (numérique)

Découvrir la revue

Citer ce compte rendu

Bélanger, L. (1992). Compte rendu de [Alan M. Glassman and Thomas G.

Cummings, Cases in Organizational Development]. Relations industrielles /

Industrial Relations, 47(1), 173-174. https://doi.org/10.7202/050756ar

Tous droits réservés (C) Département des relations industrielles de l'Université Laval, 1992
Ce document est protégé par la loi sur le droit d'auteur. L’utilisation des services d'Érudit (y compris la reproduction) est assujettie à sa politique d'utilisation que vous pouvez consulter en ligne.

https://apropos.erudit.org/fr/usagers/politique-dutilisation/ 
résultats sont présentés afin d'appliquer les notions véhiculées sur le développement des méthodes d'évaluation. Le choix de la méthode d'évaluation doit être déterminé par la raison d'être de l'évaluation. Les auteurs n'ont pas comparé les méthodes d'évaluation en fonction de: (1) leur utilisation on rapport avec les différentes activités de G.R.H.; (2) de critères quantitatís et qualitatifs d'efficacité; et (3) de leur imperméabilité à l'égard des erreurs psychométriques. Le choix de la source d'évaluation (supérieur immédiat, collègues de travail, subordonnés) ne peut être dissocié de la méthode d'évaluation. Les avantages et les inconvénients de chaque source d'évaluation sont présentés. Chacune offre une perspective particulière de la performance. Par conséquent, ces sources d'évaluation tendent à être pertinentes pour des utilisations spécifiques en évaluation. Il doit y avoir compatibilité entre la source d'évaluation et la raison d'être de l'évaluation. Les auteurs n'ont pas analysé les sources d'évaluation en fonction de la raison d'être de l'évaluation.

Le chapitre 5 décrit le processus de l'entrevue d'évaluation. Plus précisément, Boucher et Doyon discutent de la préparation, de la conduite et de la fin de l'entrevue. Par ailleurs, les auteurs traitent du style et de la motivation de l'interviewer. Ils fournissent également des recommandations quant aux comportements requis par l'interviewer. Les facteurs qui contribuent à l'efficacité de l'entrevue d'évaluation sont exposés sommairement. Les thèmes du chapitre sont analysés superficiellement. De plus, les auteurs ne discutent pas de la façon de donner du feedback négatif lors de l'entrevue et comment faire face à des situations inconfortables pendant l'évaluation.

Globalement, le livre n'est définitivement pas recommandé pour le milieu universitaire compte tenu de la faiblesse marquée du cadre théorique. Malheureusement, l'ouvrage est très incomplet pour les personnes de la pratique malgré l'intérêt que suscite le sujet.

Denis MORIN

Université Laval

Cases in Organizational Development, by Alan $M$. Glassman and Thomas G. Cummings, Homewood, IL, Richard D. Irwin, 1991, 539 p., ISBN 0256-09937-5

Issu de la dynamique de groupe et du courant des systèmes sociaux, le développement organisationnel (DO) a connu au cours des dernières années des modifications importantes au plan des cibles d'intervention sans délaisser la démarche plutôt non directive qui le caractérisait au tout début. En effet, jadis fortement centré sur l'aspect humain des organisations, le DO s'inscrit aujourd'hui dans le courant de la gestion stratégique des ressources humaines, de la culture d'entreprise en faisant de la mission, de la stratégie et des valeurs des organisations une préoccupation première au plan de lintervention.

Le présent ouvrage, qui n'est pas un traité de nature théorique, mais plutôt un recueil de cas (34 en tout), se veut un reflet des changements survenus dans ce domaine d'application des sciences du comportement et de la gestion en vue 
d'accroître la capacité des organisations de s'adapter à un environnement de moins en moins stable et prévisible.

La présentation des cas obéit à la séquence habituelle qui caractérise toute intervention auprès des organisations qui utilise l'approche DO: 1) les contacts initiaux et la nature du contrat; 2) le diagnostic organisationnel; 3) l'intervention proprement dite ou la séquence des actions porteuses d'un changement; 4) enfin, le suivi ou l'enracinement des changements effectués. La majorité des cas répertoriés viennent illustrer les étapes diagnostic et intervention qui constituent le coeur de tout programme de changement organisationnel. Des actions, de type «formation d'équipe de travail» (teambuilding), de rencontre de confrontation (confrontation me日ting), d'enquête avec rétroaction d'information aux intéressés (survey feedback), de diagnostic d'environnement externe en termes de menaces et d'opportunités sont abondamment illustrées.

Chaque cas est suivi d'une série de questions auxquelles l'étudiant en DO ou le futur intervenant cherche à apporter des réponses. C'est ainsi qu'en plus de se familiariser avec les concepts et les principes du DO, le futur consultant aura l'occasion d'acquérir les habiletés de diagnostic et de programmation d'actions pertinentes. Il faut également signaler que ces cas sont tirés à la fois de situations vécues aussi bien dans les organisations du secteur public (hôpital, centre communautaire, diocèse) que du secteur privé. De plus, les auteurs, pour la rédaction des cas, ont fait appel aux cadres supérieurs des organisations et aux consultants qui ont vécu les situations décrites. On se retrouve donc avec un premier ouvrage du genre dans le domaine du DO, alors qu'on sait que de tels recueils de cas existent depuis très longtemps dans les autres domaines de la gestion des organisations. Il s'agit donc d'un instrument précieux pour ceux et celles qui dispensent un enseignement dans ce domaine ou encore qui le pratiquent.

Laurent BÉLANGER

Université Laval

L'emploi en devenir, par Diane-Gabrielle Tremblay, Québec, Institut québécois de recherche sur la culture, collection Diagnostic, 1990, 121 p., ISBN 2-89224-141-3

La collection «Diagnostic" dans faquelle est paru le livre de Diane-Gabrielle Tremblay réunit des ouvrages portant sur des questions d'actualité et qui sont destinés au grand public. Diagnostic veut informer, provoquer la réflexion, stimuler la recherche et aider le lecteur à se former une opinion éclairée. Ce petit livre d'une centaine de pages portant sur ce que certains ont appelé les nouvelles formes d'emploi, d'autres les formes atypiques ou précaires d'emplois, remplira certainement ce rôle. Il présente, dans un langage simple, les principales formes d'emplois atypiques actuellement en progression au Québec (emplois à la pige, à temps partiel, occasionnels, à contrat, etc.) et les nouvelles théories économiques (théories de la régulation, thèses de la segmentation du marché du travail, etc.) qui tentent d'expliquer le phénomène. 